\title{
OPEN Effects of heliox and non-invasive neurally adjusted ventilatory assist (NIV-NAVA) in preterm infants
}

\begin{abstract}
Natalia Neumann-Klimasińska' ${ }^{1}$, T. Allen Merritt' ${ }^{2}$, Jennifer Beck ${ }^{3,4,5}$, Izabela Miechowicz ${ }^{6}$, Marta Szymankiewicz-Bręborowicz ${ }^{1}$ \& Tomasz Szczapa ${ }^{1 凶}$

Due to its unique properties, helium-oxygen (heliox) mixtures may provide benefits during noninvasive ventilation, however, knowledge regarding the effects of such therapy in premature infants is limited. This is the first report of heliox non-invasive neurally adjusted ventilatory assist (NIV-NAVA) ventilation applied in neonates born $\leq 32$ weeks gestational age. After baseline NIV-NAVA ventilation with a standard mixture of air and oxygen, heliox was introduced for $3 \mathrm{~h}$, followed by $3 \mathrm{~h}$ of airoxygen. Heart rate, peripheral capillary oxygen saturation, cerebral oxygenation, electrical activity of the diaphragm (Edi) and selected ventilatory parameters (e.g., respiratory rate, peak inspiratory pressure) were continuously monitored. We found that application of heliox NIV-NAVA in preterm infants was feasible and associated with a prompt and significant decrease of Edi suggesting reduced respiratory effort, while all other parameters were stable throughout the study, and had similar values during heliox and air-oxygen ventilation. This therapy may potentially enhance the efficacy of noninvasive respiratory support in preterm neonates and reduce the number of infants progressing to ventilatory failure.
\end{abstract}

Since the discovery of helium in nineteenth century, it has found numerous applications in medicine ${ }^{1}$. Its low density and diffusion coefficient make helium a particularly valuable asset in respiratory care ${ }^{2}$. According to Bernouilli's law, the low density of this gas directly affects the change in type and velocity of flow in the respiratory $\operatorname{tract}^{2}$. It may influence the respiratory function in premature infants as they are characterized by very small diameters of the airways and thus high respiratory resistance ${ }^{3}$. In the narrow airway, helium-oxygen mixture (heliox) can enhance the gas flow by reducing turbulence. In such setting, the airway pressure during ventilation with constant flow of heliox, will be lower in comparison to standard (air-oxygen) mixture ventilation. As the diffusion coefficient of carbon dioxide in the alveoli filled with helium-oxygen mixture is higher than with air, heliox ventilation can additionally improve the gas exchange ${ }^{4}$.

Conventional ventilation with heliox (helium and oxygen mixture) was found to reduce work of breathing, improve oxygenation and $\mathrm{CO}_{2}$ elimination, as well as enhance the distribution of gas within the respiratory system $^{2}$. Findings from previous studies on heliox application in newborns during mechanical ventilation are encouraging $^{5-7}$. However, knowledge about the physiological effects of this gas mixture during the non-invasive ventilation (NIV) in premature infants is limited.

Non-invasive neurally adjusted ventilatory assist (NIV-NAVA) provides a unique type of synchronization with patient's respiratory effort. The electrical activity of the diaphragm (Edi) - recorded via miniaturized sensors on the baby's nasogastric feeding tube-is used to control the timing and amplitude of assist during NAVA. The assist is achieved by adjusting the NAVA level, a conversion factor between the Edi and the delivered assist ${ }^{8}$. NIV-NAVA has been found safe and feasible, even in the most vulnerable and immature neonates ${ }^{9}$. Apart from ventilatory support NAVA enables a quantitative assessment of Edi, which can serve as a valuable diagnostic tool $^{10}$. High Edi values reflect increased respiratory effort. Provision of the adequate respiratory support results in the decrease of Edi voltage ${ }^{11}$.

\footnotetext{
${ }^{1}$ Neonatal Biophysical Monitoring and Cardiopulmonary Therapies Research Unit, Department of Neonatology, Poznan University of Medical Sciences, Poznan, ul. Polna 33, 60-535 Poznan, Poland. '2oma Linda University School of Medicine, Loma Linda, CA, USA. ${ }^{3}$ Department of Critical Care, Keenan Research Centre for Biomedical Science of St. Michael's Hospital, St. Michael's Hospital, Toronto, ON M5B1W8, Canada. ${ }^{4}$ Department of Pediatrics, University of Toronto, Toronto, Canada. ${ }^{5}$ nstitute for Biomedical Engineering and Science Technology (iBEST), St-Michael's Hospital, Ryerson University, Toronto, Canada. ${ }^{6}$ Department of Computer Science and Statistics, Poznan University of Medical Sciences, Poznan, Poland. ${ }^{\circledR}$ email: tszczapa@gmail.com
} 


\begin{tabular}{|l|l|}
\hline $\begin{array}{l}\text { Birth weight (g) } \\
\text { mean (SD) }\end{array}$ & $1396(525)$ \\
\hline $\begin{array}{l}\text { Gestational age (weeks) } \\
\text { mean (SD) }\end{array}$ & $29(3)$ \\
\hline $\begin{array}{l}\text { Day of life } \\
\text { mean (SD) }\end{array}$ & $8(8)$ \\
\hline $\begin{array}{l}\text { Apgar score 1' } \\
\text { median (range) }\end{array}$ & $6(2-9)$ \\
\hline $\begin{array}{l}\text { Apgar score 5 } \\
\text { median (range) }\end{array}$ & $8(4-9)$ \\
\hline $\begin{array}{l}\text { Multiple birth } \\
\text { n (\%) }\end{array}$ & $7(30)$ \\
\hline $\begin{array}{l}\text { Prenatal steroids } \\
\text { n (\%) }\end{array}$ & $14(61)$ \\
\hline $\begin{array}{l}\text { Chorioamnionitis } \\
\text { n (\%) }\end{array}$ & $4(17)$ \\
\hline
\end{tabular}

Table 1. Patients' characteristics.
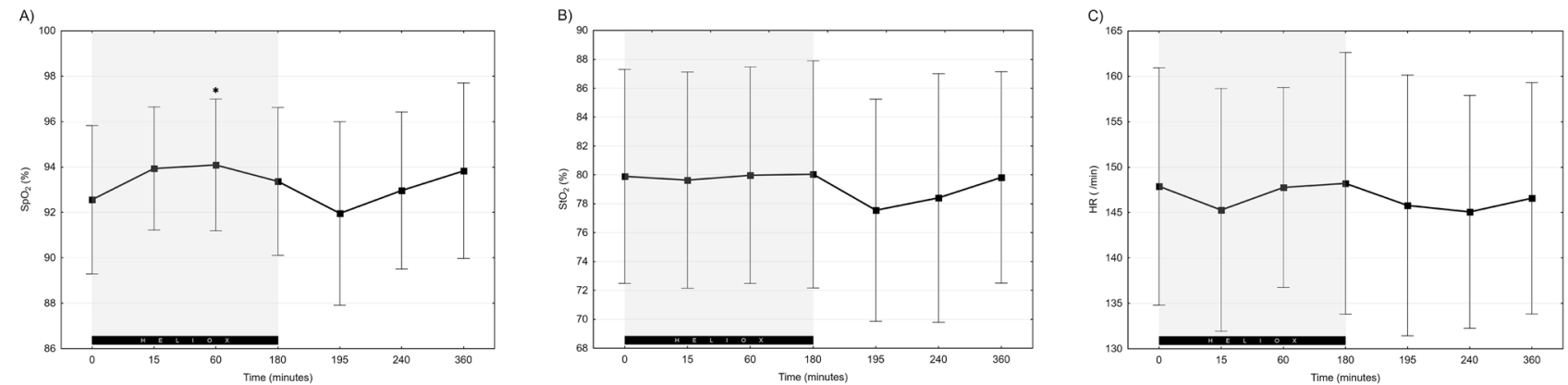

Figure 1. Values of $\mathrm{SpO}_{2}$ (A), B) $\mathrm{StO}_{2}$ (B) and $\mathrm{HR}$ (C) during the study. Grey background highlights heliox NIV-NAVA period. Data presented as mean $\pm \mathrm{SD}$; ${ }^{*}$ indicates statistically significant changes versus baseline.

Synchronization of nasal intermittent positive pressure ventilation (NIPPV) may improve outcomes in premature infants with RDS and reduce extubation failure ${ }^{12}$. In premature infants synchronized NIPPV was associated with greater decrease in the work of breathing (WOB) than nCPAP alone ${ }^{13}$. NIV-NAVA proved more efficacious than both non-synchronized NIPPV and nCPAP after extubation ${ }^{14,15}$. The combination of heliox and NIV-NAVA may promote NIV success, providing a synergistic approach to premature infants with respiratory failure. Hence, the aim of the study was to assess the influence of heliox augmented NIV-NAVA in premature infants with respiratory failure on selected physiological and clinical parameters, evaluating gas exchange and diaphragmatic function.

\section{Results}

23 premature infants born in 2017-2018 were included in the study. In 12 neonates NIV was utilized as the primary respiratory support and in 11 infants it was used post-extubation. The mean duration of pregnancy was 29 weeks GA and mean birth weight $1396 \mathrm{~g}$. The rate of antenatal steroid therapy in the studied group was low as compared with the data from the region ${ }^{16}$. However, the studied cohort included also outborn infants transferred from non-tertiary centers, possibly with reduced use of antenatal steroids. Two patients from the group receiving NIV as primary support required intubation and surfactant administration after the study. Two neonates enrolled post-extubation required reintubation within $12 \mathrm{~h}$ after the study. Table 1 presents characteristics of the studied group.

Infants' general condition was stable throughout all phases of the study. The average $\mathrm{SpO}_{2}$ in both groups ranged from 92 to $94 \%$. There was a subtle but statistically significant increase in $\mathrm{SpO}_{2}$ after 60 min of heliox ventilation ( 92.6 vs $94.1 \%, p=0.032$ ). Termination of heliox administration was associated with a small (93.4 vs 92.0\%; $p=0.389$ ), transient decrease in $\mathrm{SpO}_{2}$ (Fig. 1A). Cerebral oxygenation during the study ranged from mean $\mathrm{StO}_{2}$ of 70 to $88 \%$ (Fig. 1B). The statistical analysis of $\mathrm{StO}_{2}$ and $\mathrm{HR}$ measured in selected timepoints did not show any significant differences between study phases (Fig. 1B,C) $(p=0.156, p=0.778$, respectively).

Capillary blood gas analysis did not reveal any significant differences between study periods - mean $\mathrm{pH}$ was $7.29 \pm 0.04$ vs $7.29 \pm 0.04$ vs $7.29 \pm 0.04$ and $\mathrm{pCO}_{2} 47.4 \pm 11.6$ vs $50.0 \pm 8.8$ vs $49.6 \pm 9.5[\mathrm{~mm} \mathrm{Hg}](p=0.505)$. Ventilatory parameters and $\mathrm{FiO}_{2}$ did not differ between heliox and air-oxygen (Fig. 2A,B) $(p=0.476)$. Mean NIV leakage values were also similar $(82.18 \pm 17.17$ vs $89.27 \pm 4.92[\%], p=0.572$. NAVA level in the studied group was set between 1 and $4 \mathrm{~cm} \mathrm{H} \mathrm{H}_{2} \mathrm{O} / \mu \mathrm{V}$ (mean NAVA level $=1.55 ; \mathrm{SD}=1\left[\mathrm{~cm} \mathrm{H}_{2} \mathrm{O} / \mu \mathrm{V}\right]$ ). Heliox administration was associated with a rapid decrease in respiratory rate. After 15, 60 and $180 \mathrm{~min}$ of heliox ventilation RR was significantly lower than at beginning of the study $(p=0.017, p=0.009$ and $p=0.007$, respectively). A temporary, 

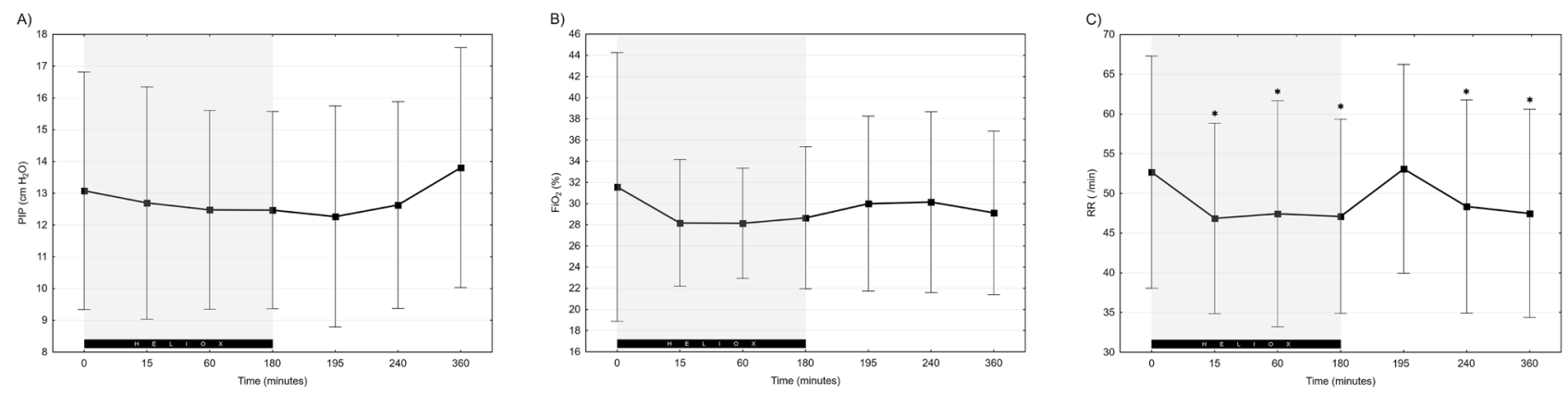

Figure 2. Ventilatory parameters during the study: $\mathrm{PIP}(\mathbf{A}), \mathrm{FiO}_{2}(\mathbf{B}), \mathrm{RR}(\mathbf{C})$. Grey background highlights heliox NIV-NAVA period. Data presented as mean \pm SD; ${ }^{*}$ indicates statistically significant changes versus baseline.
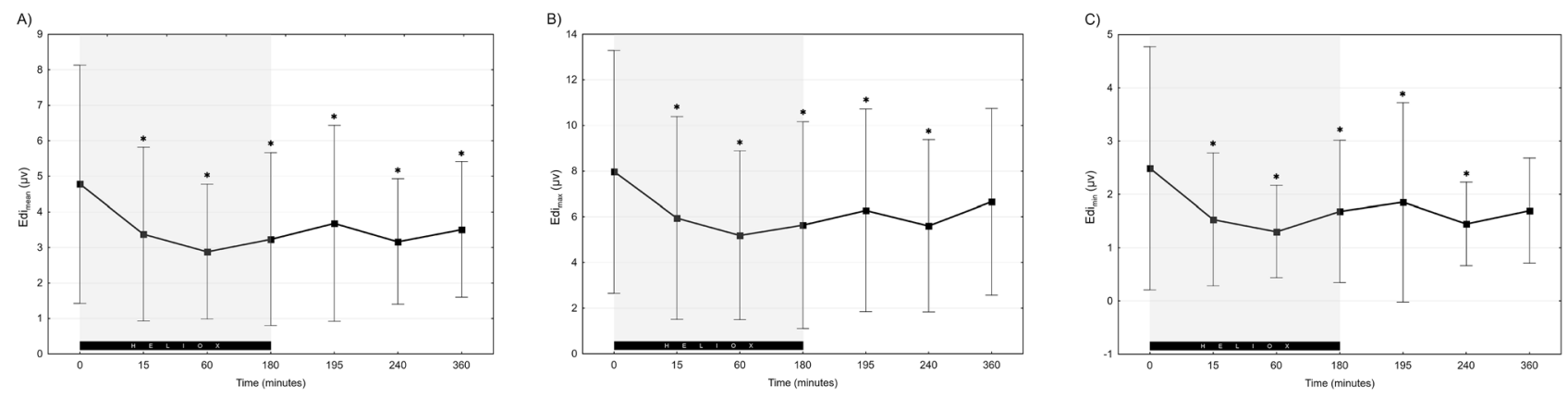

Figure 3. Electrical activity of the diaphragm during the study: $\operatorname{Edi}_{\text {mean }}(\mathbf{A}), \operatorname{Edi}_{\text {max }}(\mathbf{B}), \operatorname{Edi}_{\text {min }}(\mathbf{C})$. Grey background highlights heliox NIV-NAVA period. Data presented as mean \pm SD; *indicates statistically significant changes versus baseline.

increase in RR was observed immediately after disconnecting heliox (Fig. 2C). A significant difference was found between RR values recorded at $180 \mathrm{~min}$ of heliox and $15 \mathrm{~min}$ after return to air-oxygen ventilation (mean 47 vs 53 ; $p=0.019$ ), however after 60 and $180 \mathrm{~min}$ of air-oxygen RR decreased and did not differ significantly as compared to values before heliox termination (mean 47 vs 48 and 47 respectively; $p=0.479$ and 0.697 ).

All measures of electrical activity of the diaphragm decreased after heliox administration. After 60' of heliox particular Edi values were significantly lower than at baseline ( $\mathrm{Edi}_{\min } 1.3 \mathrm{vs} 2.5 \mu \mathrm{V}, p=0.02$; $\operatorname{Edi}_{\max } 5.2 \mathrm{vs} 8 \mu \mathrm{V}$, $p=0.002$ Edi $_{\text {mean }} 2.9$ vs $4.8 \mu \mathrm{V}, p<0.001$; Fig. $3 \mathrm{~A}$ ). $\mathrm{Edi}_{\min }$ at the beginning of the study was significantly higher than at the further timepoints, except for the value at the end of the study when Edi $\mathrm{min}_{\min }$ started to rise (Fig. $3 \mathrm{C}$ ). $\mathrm{Edi}_{\text {mean }}$ and $\mathrm{Edi}_{\text {min }}$ at the end of the protocol were significantly higher than in the $60^{\text {th }}$ minute after heliox NIVNAVA (3.5 vs $2.9[\mu \mathrm{V}], p=0.01 ; 1.7$ vs $1.3[\mu \mathrm{V}] p=0.03)$. However, the last recorded Edi $\mathrm{i}_{\text {mean }}$ was significantly lower than values observed at baseline ( 4.8 vs $3.5[\mu \mathrm{V}], p=0.024$ ) (Fig. 3B).

In the subgroup analysis of primary heliox NIV-NAVA patients Edi $_{\text {mean }}$ differed significantly from baseline at 60 and $180 \mathrm{~min}$ of heliox-3.87 vs 2.57 and $2.83[\mu \mathrm{V}]$, respectively $(p=0.008)$. Similar trends in Edi values were found in the post extubation subgroup, however observed differences were not significant.

Edi $_{\max }$ was significantly lower during heliox NIV-NAVA in comparison to the initial value. Discontinuation of heliox was associated with slow increase in $\mathrm{Edi}_{\max }$, which at the 360th minute of the study was significantly higher than in the $60^{\text {th }}$ and $180^{\text {th }}$ minute (6.7 vs 5.2 and $5.6[\mu \mathrm{V}]$ respectively; $p=0.006, p=0.02$ ).

\section{Discussion}

Ensuring the success of NIV in premature infants remains a major challenge to modern neonatology, as a considerable number of infants born prematurely fail early nasal continuous positive airway pressure (nCPAP) $)^{17,18}$. Once on mechanical ventilation, weaning may also present a difficulty, especially in the setting of developing bronchopulmonary dysplasia ${ }^{19}$. Heliox administration during non-invasive ventilation has been evaluated in a limited number of studies but it has been shown that it may be associated with certain clinical benefits. When combined with nCPAP, heliox was found to reduce the need for mechanical ventilation in preterm infants with respiratory distress syndrome in comparison with standard nCPAP ${ }^{20}$. Delivered with non-synchronized nasal intermittent positive pressure ventilation (NIPPV), heliox was effective in reducing the length of ventilation and increasing carbon dioxide elimination ${ }^{21}$. To our knowledge this is the first study assessing effects of heliox during NIV-NAVA in premature infants.

Few studies have assessed the effectiveness of heliox therapy on respiratory effort. Wolfson et al. reported that infants with bronchopulmonary dysplasia receiving heliox via face mask responded with a reduction in work of breathing (WOB) as measured by simultaneous pneumotachography and esophageal pressure ${ }^{22}$. A decrease in $\mathrm{WOB}$ was also revealed during heliox augmented mechanical ventilation ${ }^{23}$ and heliox delivery via nasal 
cannulae ${ }^{24}$. The present study confirms these observations using a new methodology, considering Edi as a surrogate for the WOB measurement. A study in an animal model found that increased diaphragm load, expressed by high voltage $\mathrm{Edi}_{\max }$, is associated with damage to its sarcomeres ${ }^{25}$. In a porcine model of acute lung injury, high flow nasal cannula (HFNC) with heliox demonstrated a protective effect towards the diaphragm ${ }^{26}$. The significant and rapid decrease of Edi voltage after introduction of heliox suggests that the change of breathing mixture to heliox has direct influences on respiratory function. Absolute Edi values may be difficult to compare between different infants due to high variability, hence trends of this parameter seem most useful for assessment of changes in respiratory effort.

As potential rapid changes in partial pressure of $\mathrm{CO}_{2}$ due to improved gas flow and diffusion might influence cerebral blood flow $\mathrm{StO}_{2}$ monitoring during heliox with NIV-NAVA was performed. In this study no significant changes in $\mathrm{StO}_{2}$ were observed during heliox ventilation. However, patients included in the study were relatively stable and their baseline $\mathrm{StO}_{2}$ was within the normal range. Future studies should determine whether heliox might help to improve low $\mathrm{StO}_{2}$ in patients with severe respiratory failure. Although there are studies on heliox MV only single publications report on non-invasive respiratory support and the influence of the therapy on oxygenation in infants $s^{27,28}$. In the presented study the increase in $\mathrm{SpO}_{2}$ values after $1 \mathrm{~h}$ of heliox was statistically significant but very subtle-most likely of limited clinical importance. Withdrawal of heliox was associated with a significant decrease in $\mathrm{SpO}_{2}$, which confirms earlier observations reported by our group ${ }^{3-5}$.

In previous studies discontinuation of heliox after $1 \mathrm{~h}$ of mechanical ventilation was associated with significant deterioration of oxygenation and respiratory function suggesting that the duration of therapy might have been too short $^{3-5}$. In the study by Dani et al., a decrease in $\mathrm{MAP}$ and $\mathrm{pCO}_{2}$ was found after $24 \mathrm{~h}$ of heliox ${ }^{29}$. Colnaghi et al. reported a reduction of non-invasive ventilation failure risk after 12-h of NIV with mixture of helium and oxygen ${ }^{17}$. We found the effect of heliox on Edi was observed after $15 \mathrm{~min}$ and the effect on $\mathrm{SpO}_{2}$ after one hour. After heliox NIV-NAVA was discontinued there was a trend towards return to pre-heliox Edi values. However, they did not reach the pre-intervention value, suggesting an improvement of respiratory function. The question remains whether prolonging the treatment with heliox beyond $3 \mathrm{~h}$ would further improve the clinical effects of the therapy. The minimum duration of successful treatment with neonatal heliox remains unknown and will likely depend on the underlying disease and the individual condition of each patient. The challenge in future studies will be to determine the threshold parameters for ending heliox therapy without the risk of deterioration. Edi decrement could serve as a potential predictor of the success of heliox therapy.

Application of NIV is associated with leak which can be influenced by the type of the interface used and its fitting. Based on theoretical assumptions regarding physical properties of heliox (low density and high diffusion coefficient) its use during NIV might be associated with increased leak as compared to air-oxgen. However, no significant difficulties regarding leak were observed in this study when heliox was delivered with NIV-NAVA using nasal masks. Effects of heliox were also described when it was used with high flow therapy during which high leak is routinely expected ${ }^{23}$. High leak during NIV makes flow-triggered synchronization challenging, however while Edi is used for triggering this is not a problem with NIV-NAVA.

Limitations of this pilot study include a small number of participants with relatively modest respiratory requirements and two types of NIV application: primary and post-extubation. Study protocol was not blinded, leading to a potential bias. Moreover, only selected physiologic parameters were analyzed over limited period of time, hence it is not possible to speculate about the long-term improvements in respiratory function or the impact of therapy on pulmonary outcomes. As suggested before there may be a synergistic effect of heliox and NIV-NAVA and it is difficult to assess their contribution to the observed effects. However, as NAVA level was kept constant during the study, decreasing Edi values after heliox administration and their increase observed following heliox discontinuation strongly suggest the influence of the gas mixture on the respiratory function.

Presented results of this pilot study warrant further research in a larger group of patients to better understand the potential synergistic effect of heliox and NIV-NAVA and the role of this combined therapy in enhancing the efficacy of non-invasive respiratory support in the preterm infants.

\section{Methods}

An unblinded crossover pilot study was conducted in the NICU of the Department of Neonatology, Poznan University of Medical Sciences in Poznan, Poland. Infants eligible for inclusion were born $\leq 32$ weeks gestational age (GA), had respiratory distress and required NIV as primary ventilation with fraction of inspired oxygen $\left(\mathrm{FiO}_{2}\right)$ between 0.25 and 0.4 or secondary, after at least one failed extubation attempt. The latter were assessed by the treating physician as ready for another extubation according to specific criteria (spontaneous respiratory drive, mean airway pressure (MAP) $<8 \mathrm{~cm} \mathrm{H}_{2} \mathrm{O}$, capillary $\mathrm{pH}>7.22$ and $\mathrm{pCO}_{2}<60 \mathrm{mmHg}$, post caffeine citrate administration); they received heliox NIV-NAVA within $1 \mathrm{~h}$ from extubation. Patients with major congenital defects, respiratory failure requiring mechanical ventilation or rescue surfactant administration were excluded from the trial. The study protocol was approved by the ethics committee of Poznan University of Medical Sciences. For all eligible infants written informed consent was obtained from their parents. The study has been performed in accordance with the Declaration of Helsinki and registered at ClinicalTrials.gov (NCT04404816, 28/05/2020).

Premature infants were first switched from non-synchronized NIPPV to NIV-NAVA using Maquet Servo-I ventilator (Getinge, Solna, Sweden). In the initial phase of the study NAVA level was optimized aiming at achieving peak inspiratory pressure (PIP) similar to values during preceding NIPPV, with positive end-expiratory pressure (PEEP) set at $5 \mathrm{~cm} \mathrm{H}_{2} \mathrm{O}$. When this was achieved recordings of $30 \mathrm{~min}$ were acquired at baseline while infants were ventilated with air-oxygen NIV-NAVA. Afterwards air was switched to heliox-mixture of $79 \%$ helium and $21 \%$ oxygen (Linde Group, Poland). After $3 \mathrm{~h}$ heliox was discontinued and the gas supply was changed back to the standard air-oxygen mixture. Ventilatory parameters, including NAVA level, were kept constant throughout the study. $\mathrm{FiO}_{2}$ was titrated to keep the $\mathrm{SpO}_{2}$ in the range of $90-95 \%$. Monitoring of cerebral oxygenation 
$\left(\mathrm{StO}_{2}\right)$, pulse oximeter oxygen saturation $\left(\mathrm{SpO}_{2}\right)$ and heart rate $(\mathrm{HR})$ were performed throughout the study using NONIN SenSmart Model X-100 (Nonin Medical Inc., Plymouth, USA). Selected ventilation parameters (PIP, PEEP, MAP, RR, NIV leakage), as well as Edi measures were recorded using Servo-tracker software (Getinge, Solna, Sweden). Blood gas analysis was performed in 3 study periods-at baseline, $3 \mathrm{~h}$ after heliox administration and $3 \mathrm{~h}$ after the return to standard gas mixture (Cobas b 221 blood gas system, Roche, Switzerland).

Analyses were performed using Statistica 12 (TIBCO Software, Palo Alto, California, USA) and PQStat v.1.6.8 software (PQStat Software, Poznan, Poland); $p<0.05$ was considered significant. The normal distribution of variables was checked using the Shapiro-Wilk test. In order to examine changes in time, in the case of compliance with the normal distribution and equal variances, the variance analysis test was calculated for related samples. In the absence of compliance with a normal distribution the Friedman test was used. In the case of statistically significant differences in order to assess between which timepoints they occurred post hoc, Conover-Iman's test was performed.

The datasets generated during and/or analyzed during the current study are available from the corresponding author on request.

Received: 29 March 2021; Accepted: 23 July 2021

Published online: 04 August 2021

\section{References}

1. Hess, D. R. et al. The history and physics of heliox. Respir. Care 51(6), 608-612 (2006).

2. Kass, J. E. Heliox redux. Chest 123(3), 673-676 (2003).

3. Myers, T. R. Use of heliox in children. Respir. Care 51(6), 619-631 (2006).

4. Venkataraman, S. T. Heliox during mechanical ventilation. Respir. Care 51(6), 632-639 (2006).

5. Szczapa, T. \& Gadzinowski, J. Use of heliox in the management of neonates with meconium aspiration syndrome. Neonatology 100(3), 265-270 (2011).

6. Szczapa, T., Gadzinowski, J. \& Moczko, J. Heliox augmented mechanical ventilation in the treatment of premature infants with respiratory distress syndrome. Ginekol. Pol. 85(12), 939-943 (2014).

7. Szczapa, T., Gadzinowski, J., Moczko, J. \& Merritt, T. A. Heliox for mechanically ventilated newborns with bronchopulmonary dysplasia. Arch. Dis. Childhood Fetal Neonatal. Edn. 99(2), F128-F133 (2014).

8. Sinderby, C. et al. Neural control of mechanical ventilation in respiratory failure. Nat. Med. 5(12), 1433-1436 (1999).

9. Gibu, C. K., Cheng, P. Y., Ward, R. J., Castro, B. \& Heldt, G. P. Feasibility and physiological effects of noninvasive neurally adjusted ventilatory assist in preterm infants. Pediatr. Res. 82(4), 650-657 (2017).

10. Stein, H., Hall, R., Davis, K. \& White, D. B. Electrical activity of the diaphragm (Edi) values and Edi catheter placement in nonventilated preterm neonates. J. Perinatol. 33(9), 707-711 (2013).

11. Firestone, K. S., Fisher, S., Reddy, S., White, D. B. \& Stein, H. M. Effect of changing NAVA levels on peak inspiratory pressures and electrical activity of the diaphragm in premature neonates. J. Perinatol. 35(8), 612-616 (2015).

12. Sweet, D. G. et al. European consensus guidelines on the management of respiratory distress syndrome-2019 update. Neonatology 115(4), 432-450 (2019).

13. Aghai, Z. H. et al. Synchronized nasal intermittent positive pressure ventilation (SNIPPV) decreases work of breathing (WOB) in premature infants with respiratory distress syndrome (RDS) compared to nasal continuous positive airway pressure (NCPAP). Pediatr. Pulmonol. 41(9), 875-881 (2006).

14. Yagui, A. C, Gonçalves, P. A., Murakami, S. H., Santos, A. Z., Zacharias, R. S. B., \& Rebello, C. M. Is noninvasive neurally adjusted ventilatory assistance (NIV-NAVA) an alternative to NCPAP in preventing extubation failure in preterm infants? J. Maternal-Fetal Neonatal Med. (2019).

15. Makker, K. et al. Comparison of extubation success using noninvasive positive pressure ventilation (NIPPV) versus noninvasive neurally adjusted ventilatory assist (NI-NAVA). J. Perinatol. 40, 1202-1210 (2020).

16. Zeitlin, J., Manktelow, B. N., Piedvache, A., Cuttini, M., Boyle, E., \& van Heijst, A., et al. Use of evidence based practices to improve survival without severe morbidity for very preterm infants: results from the EPICE population based cohort. BMJ 354 (2016)

17. Gulczyńska, E., Szczapa, T., Hożejowski, R., Borszewska-Kornacka, M. K. \& Rutkowska, M. Fraction of inspired oxygen as a predictor of CPAP failure in preterm infants with respiratory distress syndrome: A prospective multicenter study. Neonatology 116(2), 171-178 (2019).

18. Dargaville, P. A., Gerber, A., Johansson, S., de Paoli, A. G., Kamlin, C. O. F, \& Orsini, F., et al. Incidence and outcome of CPAP failure in preterm infants. Pediatrics. 138(1) (2016).

19. Manley, B. J., Doyle, L. W., Owen, L. S. \& Davis, P. G. Extubating extremely preterm infants: Predictors of success and outcomes following failure. J. Pediatr. 173, 45-49 (2016).

20. Colnaghi, M. et al. Nasal continuous positive airway pressure with heliox in preterm infants with respiratory distress syndrome. Pediatrics 129(2), e333-e338 (2012).

21. Li, X., Shen, J., Zhao, J., Tang, S. \& Shi, Y. Nasal intermittent positive pressure ventilation with heliox in premature infants with respiratory distress syndrome: A randomized controlled trial. Indian Pediatr. 51(11), 900-902 (2014).

22. Wolfson, M. R., Bhutani, V. K., Shaffer, T. H. \& Bowen, F. W. Mechanics and energetics of breathing helium in infants with bronchopulmonary dysplasia. J. Pediatr. 104, 752 (1984).

23. Migliori, C., Gancia, P., Garzoli, E., Spinoni, V. \& Chirico, G. The effects of helium/oxygen mixture (heliox) before and after extubation in long-term mechanically ventilated very low birth weight infants. Pediatrics 123(6), 1524-1528 (2009).

24. Williams, J., Stewart, K., Tobias, J. D. \& Berkenbosch, J. W. Therapeutic benefits of helium-oxygen delivery to infants via nasal cannula. Pediatr. Emerg. Care 20(9), 574-578 (2004).

25. Shimatani, T., Shime, N., Nakamura, T., Ohshimo, S., Hotz, J., \& Khemani, R. G. Neurally adjusted ventilatory assist mitigates ventilator-induced diaphragm injury in rabbits. Respir. Res. 20(1) (2019).

26. Jassar, R. K. et al. High flow nasal cannula (HFNC) with heliox decreases diaphragmatic injury in a newborn porcine lung injury model. Pediatr. Pulmonol. 49(12), 1214-1222 (2014).

27. Martinón-Torres, F., Rodríguez-Núñez, A. \& María, M.-Sánchez. J. Nasal continuous positive airway pressure with heliox in infants with acute bronchiolitis. Respir. Med. 100(8), 1458-1462 (2006).

28. Seliem, W. \& Sultan, A. M. Heliox delivered by high flow nasal cannula improves oxygenation in infants with respiratory syncytial virus acute bronchiolitis. J. Pediatr. 94(1), 56-61 (2018).

29. Dani, C., Fontanelli, G., Lori, I., Favelli, F. \& Poggi, C. Heliox non-invasive ventilation for preventing extubation failure in preterm infants. J. Maternal-Fetal Neonatal. Med. 26(6), 603-607 (2013). 


\section{Author contributions}

T.S., T.A.M. and N.N. designed the study. JB consulted methodology. N.N. and T.S. enrolled patients and conducted data acquisition. N.N., T.S. and I.M. analyzed the results. N.N., T.A.M. and T.S. wrote the main manuscript text. I.M. prepared Figs. 1-3. MSB and TS supervised the project. All authors reviewed the manuscript.

\section{Funding}

The study was supported by European Society for Pediatric Research Young Investigator START-UP Award 2018.

\section{Competing interests}

Dr. Beck has made inventions related to neural control of mechanical ventilation that are patented. The patents are assigned to the academic institution(s) where inventions were made. The license for these patents belongs to Maquet Critical Care. Future commercial uses of this technology may provide financial benefit to Dr. Beck through royalties. Dr Beck owns $50 \%$ of Neurovent Research Inc (NVR). NVR is a research and development company that builds the equipment and catheters for research studies. NVR has a consulting agreement with Maquet Critical Care. Other authors declare no conflict of interest.

\section{Additional information}

Correspondence and requests for materials should be addressed to T.S.

Reprints and permissions information is available at www.nature.com/reprints.

Publisher's note Springer Nature remains neutral with regard to jurisdictional claims in published maps and institutional affiliations.

(c) Open Access This article is licensed under a Creative Commons Attribution 4.0 International License, which permits use, sharing, adaptation, distribution and reproduction in any medium or format, as long as you give appropriate credit to the original author(s) and the source, provide a link to the Creative Commons licence, and indicate if changes were made. The images or other third party material in this article are included in the article's Creative Commons licence, unless indicated otherwise in a credit line to the material. If material is not included in the article's Creative Commons licence and your intended use is not permitted by statutory regulation or exceeds the permitted use, you will need to obtain permission directly from the copyright holder. To view a copy of this licence, visit http://creativecommons.org/licenses/by/4.0/.

(C) The Author(s) 2021 